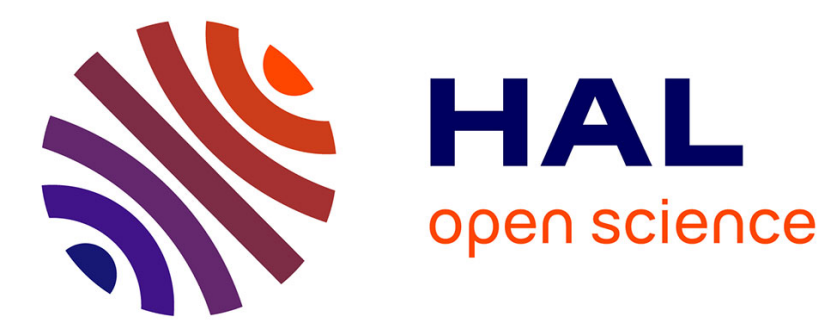

\title{
Periodic Orbits Close to Grazing for an Impact Oscillator
}

David Chillingworth, Arne Nordmark

\section{To cite this version:}

David Chillingworth, Arne Nordmark. Periodic Orbits Close to Grazing for an Impact Oscillator. Recent Trends in Dynamical Systems, pp.25-37, 2013, Recent Trends in Dynamical Systems, 10.1007/978-3-0348-0451-6_2 . hal-01508933

\section{HAL Id: hal-01508933 \\ https://hal.science/hal-01508933}

Submitted on 18 Apr 2017

HAL is a multi-disciplinary open access archive for the deposit and dissemination of scientific research documents, whether they are published or not. The documents may come from teaching and research institutions in France or abroad, or from public or private research centers.
L'archive ouverte pluridisciplinaire HAL, est destinée au dépôt et à la diffusion de documents scientifiques de niveau recherche, publiés ou non, émanant des établissements d'enseignement et de recherche français ou étrangers, des laboratoires publics ou privés. 


\title{
Periodic Orbits Close to Grazing for an Impact Oscillator
}

\author{
D.R.J. Chillingworth and A.B. Nordmark \\ D.R.J. Chillingworth \\ Mathematical Sciences, University of Southampton, Southampton SO17 1BJ, UK \\ e-mail:drjc@soton.ac.uk
}

\author{
A.B. Nordmark \\ Department of Mechanics, KTH, 10044 Stockholm, Sweden \\ e-mail: nordmark@mech.kth.se
}

\begin{abstract}
We show how the geometric impact surface approach to the dynamics of an impact oscillator provides an immediate visualization of the criteria that determine the existence of an impacting periodic orbit close to grazing. We recover the criteria set out earlier by A. Nordmark and indicate how the geometric setting and singularity geometry may be exploited to yield appropriate criteria in degenerate situations where the Nordmark criteria would not apply.
\end{abstract}

\subsection{The Impact Oscillator}

There are many physical and engineering contexts in which a vibrating or oscillating system encounters impacts-typically accompanied by undesirable noise and wear and thus demanding to be eliminated or at least controlled. See e.g. [1,3,14], as well as [9], for examples and further references.

The simplest model for such a system is the one degree of freedom impact oscillator of the form

$$
\ddot{x}+f(x, \dot{x})=g(t)
$$

where $f: \mathbf{R}^{2} \rightarrow \mathbf{R}$ and $g: \mathbf{R} \rightarrow \mathbf{R}$ are smooth functions, $g$ is $T$-periodic with $T>0$, and where $x$ is restricted to the region $x \geq c$. To describe the local 
consequence of impact with the obstacle at $x=c$ a restitution law is assumed, which usually takes the form of replacing $\dot{x}<0$ by $-r \dot{x}, r>0$ at impact.

Impact oscillators can be seen as special cases of $N$-degree of freedom (autonomous) systems with codimension-1 obstacles, or of general hybrid systems involving piecewise-smooth vector fields in $\mathbf{R}^{n}$ together with maps defined on the codimension-1 boundaries of the regions of smoothness: see [1] for further discussion.

An impact oscillator (2.1) exhibits certain bifurcation phenomena that arise specifically from the impacts, in addition to those seen in smooth (non-impacting) systems. The most natural example to study first is that in which a hyperbolic (or, at least, isolated and persistent) periodic orbit of period $T$ lying in the region $x>c$ collides with the obstacle at $x=c$ as a parameter $\mu$ in the system increases through a critical value $\mu_{0}$. For $\mu=\mu_{0}$ the orbit satisfies $\dot{x}=0$ where $x=c$, that is it grazes the obstacle. It is not a priori clear whether such a periodic orbit will persist (with impacts) for $\mu>\mu_{0}$ or how the result may depend on the nature of the Poincaré map of the grazing orbit.

There have been many studies, both theoretical and numerical, of the dynamical behaviour of an impact oscillator close to grazing: see for example [2, 4, 6-8, 10 $12,15,16]$. A thorough investigation of the persistence or otherwise of a grazing $T$-periodic orbit was carried out by Nordmark in [13] in the wider context of an $n$-degree of freedom (autonomous) system with a codimension- 1 obstacle, and as an illustration in that paper the general results are applied to the 1-degree of freedom system (2.1). A detailed analysis of criteria for the persistence of orbits of period $n T$ for $n=1,2,3 \ldots$ is carried out, leading to interesting algebra related to the eigenvalues of the Poincaré map. However, simple criteria in terms of the Poincaré map itself are not immediately visible.

The purpose of this paper is, first, to extract the essential data from the general formalism of [13] in order to write down what is needed for the impact oscillator (2.1) in the cases $n=1$ and $n=2$. Having done this, we then take a different approach and use the impact surface description formulated in [5] in order to reveal in this setting the extremely simple geometric criteria that underlie the phenomenon of grazing bifurcation. Finally we show how this geometry, when converted into algebra, yields the Nordmark criteria.

\subsubsection{Nordmark's Criteria}

We apply the general formalism from [13] to this setting, and in particular to the cases designated there as $(n)$ where $n=1$ or $n=2$, that is cases of impacting orbits close to grazing that have a single impact during a period of $T$ or of $2 T$. The aim is to derive simple criteria for the existence of such periodic orbits as a system with a grazing orbit $P$ of period $T$ is perturbed by a parameter $\mu$. We suppose without loss of generality that the grazing occurs when $\mu=0$. 


\subsubsection{Single Impact $T$-Periodic Orbits}

Following the notation from [13], let

$$
A=\left(\begin{array}{ll}
\alpha & \gamma \\
\beta & \delta
\end{array}\right)
$$

denote the Jacobian matrix of the time- $T$ map in the $(x, \dot{x})$-plane for the system (2.1) (in the absence of the obstacle) at the point $p=(c, 0)$ when $\mu=0$. The $T$-periodicity of $P$ means that $p$ is a fixed point of the map. It is convenient to introduce also the covector $C=[1,0]$ and the vector $B=[0,1]^{t}$ where ${ }^{t}$ denotes transpose. ${ }^{1}$

First assume that

$$
\Delta_{-}:=\operatorname{det}(I-A) \neq 0
$$

so that $P$ (although not necessarily hyperbolic) persists as a $T$-periodic orbit $P_{\mu}$ in the absence of the obstacle for small $|\mu|>0$. A central role in [13] is played by the quantity

$$
\begin{aligned}
e: & =C(I-A)^{-1}(\kappa, \lambda)^{t} \\
& =\Delta_{-}^{-1}((1-\delta) \kappa+\gamma \lambda)
\end{aligned}
$$

where $(\kappa, \lambda)=\left(\frac{\partial x}{\partial \mu}, \frac{\partial \dot{x}}{\partial \mu}\right)$ evaluated at $p$ when $\mu=0$.

To first order in $\mu$, the location of a $T$-periodic $(n=1)$ single-impacting orbit (when it exists) relative to the grazing point $p$ is given by $\mu X \in \mathbf{R}^{2}$ where ([13, eq. (51)])

$$
C(I-A) X=0, \quad C X=-1
$$

and from [13, eq. (34)] the condition for such an orbit to exist is

$$
y_{1} e \mu>0
$$

where from [13, eq. (32)] we have (up to a positive multiple)

$$
y_{1}=B^{t}(I-A) X \text {. }
$$

\footnotetext{
${ }^{1}$ The vector $B$ differs from that in [13] by a positive scalar multiple.
} 
From the second equation of (2.4) we see $X$ has the form $X=\left(-1, x_{1}\right)^{t}$, and then combining (2.4) and (2.6) we may write

$$
(I-A)\left(\begin{array}{cc}
-1 & 0 \\
x_{1} & 1
\end{array}\right)=\left(\begin{array}{cc}
0 & -\gamma \\
y_{1} & 1-\delta
\end{array}\right)
$$

which on taking determinants gives

$$
\gamma y_{1}=-\Delta_{-}
$$

Assuming $\gamma \neq 0$ this gives

$$
y_{1} e=-\gamma^{-1}((1-\delta) \kappa+\gamma \lambda)
$$

and so the criterion (2.5) gives the following basic result:

Proposition 2.1 (Nordmark). Suppose $\Delta_{-} \neq 0, \gamma \neq 0$ and also

$$
\psi:=(1-\delta) \kappa+\gamma \lambda \neq 0
$$

For $|\mu|$ sufficiently small, a single-impact orbit of period T exists close to the grazing orbit if and only if the parameter $\mu$ has the opposite sign from $\gamma \psi$.

In particular this implies that as $\mu$ passes through zero an impacting period- $T$ orbit must exist on one side or the other. If it exists for $\mu>0$ then the impacting orbit is a continuation of the non-impacting orbit, while for the case $\mu<0$ the free and the impacting orbit exist simultaneously and mutually annihilate at $\mu=0$. The latter phenomenon is often called a nonsmooth fold: see [1].

\subsubsection{Single Impact 2T-Periodic Orbits}

For a $2 T$-periodic orbit ( $n=2$ ) with single impact the location $X$ is now by analogy with (2.4) given by

$$
C\left(I-A^{2}\right) X=0, \quad C X=-1
$$

while the conditions for such an orbit to exist are

$$
\begin{array}{r}
b_{2,1} \text { e } \mu>0 \\
y_{2} \text { e } \mu>0
\end{array}
$$

where

$$
\begin{aligned}
b_{2,1} & =C A X+1 \\
y_{2} & =B^{t}\left(I-A^{2}\right) X .
\end{aligned}
$$


Let $\alpha_{2}, \gamma_{2}$ denote the $\alpha, \gamma$-entries in $A^{2}$, that is

$$
\begin{aligned}
& \alpha_{2}=\alpha^{2}+\beta \gamma=\alpha \eta-\operatorname{det} A \\
& \gamma_{2}=\gamma \eta
\end{aligned}
$$

where $\eta=\operatorname{tr} A$ is the trace of $A$. By analogy with (2.7) we have on taking determinants

$$
\eta \gamma y_{2}=-\operatorname{det}\left(I-A^{2}\right)=-\Delta_{-} \Delta_{+}
$$

with $\Delta_{+}=\operatorname{det}(I+A)$, and so

$$
\gamma y_{2} e=-\eta^{-1} \Delta_{+}((1-\delta) \kappa+\gamma \lambda)
$$

provided $\eta \neq 0$. If (2.11) holds then the condition (2.10) can simply be expressed by saying that $b_{2,1}$ and $y_{2}$ have the same sign. Now if $X=\left(-1, x_{2}\right)^{t}$ satisfies (2.9) we find

$$
\begin{aligned}
b_{2,1} & =-\alpha+\gamma x_{2}+1 \\
& =-\alpha+\gamma \gamma_{2}^{-1}\left(\alpha_{2}-1\right)+1 \\
& =-\eta^{-1} \Delta_{-}
\end{aligned}
$$

using (2.14) and (2.15). From (2.16) the same-sign condition on $b_{2,1}$ and $y_{2}$ is therefore precisely the condition that $\gamma \Delta_{+}>0$. To summarize:

Proposition 2.2 (Nordmark). Suppose $\Delta_{-} \neq 0, \eta \psi \neq 0$ and also $\gamma \Delta_{+} \neq 0$. For $|\mu|$ sufficiently small, a single-impact orbit of period $2 T$ exists close to the grazing orbit if and only if $\gamma \Delta_{+}>0$ and $\mu$ has the opposite sign from $\eta \psi$.

\subsubsection{The Impact Surface Approach}

We now turn to address the same questions, but from the point of view of the geometry of the impact surface as set out in [5]. We briefly describe the formalism, and refer to [5] for further details.

The impact surface $V_{c}$ is defined as

$$
V_{c}=\left\{(\tau, v, t) \in \mathbf{R}^{3}: x_{c}(\tau, v, t)=c\right\}
$$

where $x(c, v, \tau ; u)$ denotes the solution of (2.1) with initial data $(x, \dot{x})=(c, v)$ when $u=\tau$, and we write $x_{c}(\tau, v, t)=x(c, v, \tau ; \tau+t)$. 
Clearly $V_{c}$ contains the $t=0$ plane $\Pi$, and in [5] it is shown that $V_{c}=\Pi \cup V_{c}^{\prime}$ where $V_{c}^{\prime}$ is a smooth 2-manifold (except generically for finitely many values of $c$ at which $V_{c}^{\prime}$ undergoes a Morse perestroika) that intersects $\Pi$ transversely along the $\tau$-axis. The impacting system can then be modelled by a discontinuous discrete dynamical system on $\Pi$ defined as the composition

$$
G_{c}:=R \circ \phi_{c} \circ F_{c}: \Pi \rightarrow \Pi
$$

where

$F_{c}: \Pi \rightarrow V_{c}^{\prime}$ is the first hit map $(\tau, v) \mapsto\left(\tau, v, t_{1}\right)$ where $t_{1}$ is the smallest positive value of $t$ for which $(\tau, v, t) \in V_{c}^{\prime}$

$\phi_{c}: V_{c}^{\prime} \rightarrow \Pi$ is the reset map $(\tau, v, t) \mapsto(\tau+t, \dot{x}(\tau+t))$

$R: \Pi \rightarrow \Pi$ is the restitution map (such as $(\tau, v) \mapsto(\tau,-r v)$ ).

In other words, we reconstruct the dynamics by taking each impact at $x=c$ as a new set of initial data and then proceeding (after applying restitution) to the next impact.

Remark 2.1. In general the map $F_{c}$ may fail to be defined for either of the following two reasons: for given $(\tau, v) \in \Pi$ the subset $\left\{t:(\tau, v, t) \in V_{c}^{\prime}\right\}$ of $\mathbf{R}$ may be empty, or (when $v=0$ ) may accumulate at zero. Neither of these arises in the present discussion, however. It is also the case that infinitely many impacts can occur in a finite time interval (the chatter phenomenon), but again for current purposes this does not concern us.

\subsubsection{Singularity Geometry}

The singularity structure of the projection map

$$
\pi_{c}=\pi \mid V_{c}^{\prime}:(\tau, v, t) \mapsto(\tau, v)
$$

and of the reset map $\phi_{c}$ are crucial in construction of the dynamical map $G_{c}: \Pi \rightarrow \Pi$. For $(\tau, 0, t) \in V_{c}^{\prime}$ let $a=a(\tau):=\ddot{x}_{c}(\tau, 0,0)$ denote the 'initial' acceleration. Then from [5] we have

\section{Proposition 2.3.}

1. The singular set $S\left(\pi_{c}\right)$ consists of the points on $V_{c}^{\prime}$ where $\dot{x}_{c}=0$. If $\ddot{x}_{c} \neq 0$ then the singularity is a fold singularity.

2. The singular set $S\left(\phi_{c}\right)$ consists of the points on $V_{c}^{\prime}$ where $v=0$. If $a \neq 0$ then the singularity is a fold singularity.

The first part of the proposition is elementary, from the geometry of $V_{c}^{\prime}$ itself, although the second part is less obvious. Note that $\pi_{c} S\left(\pi_{c}\right)$ is the apparent outline (or contour) of $V_{c}^{\prime}$ as viewed along the $t$-axis; in [5] we call $S\left(\pi_{c}\right)$ the horizon curve $H$. 
Fig. 2.1 Two fold maps $\pi_{c}, \phi_{c}: V_{c}^{\prime} \rightarrow \Pi$. The point $p_{1} \in V_{c}^{\prime}$ is a singular (fold) point for both maps

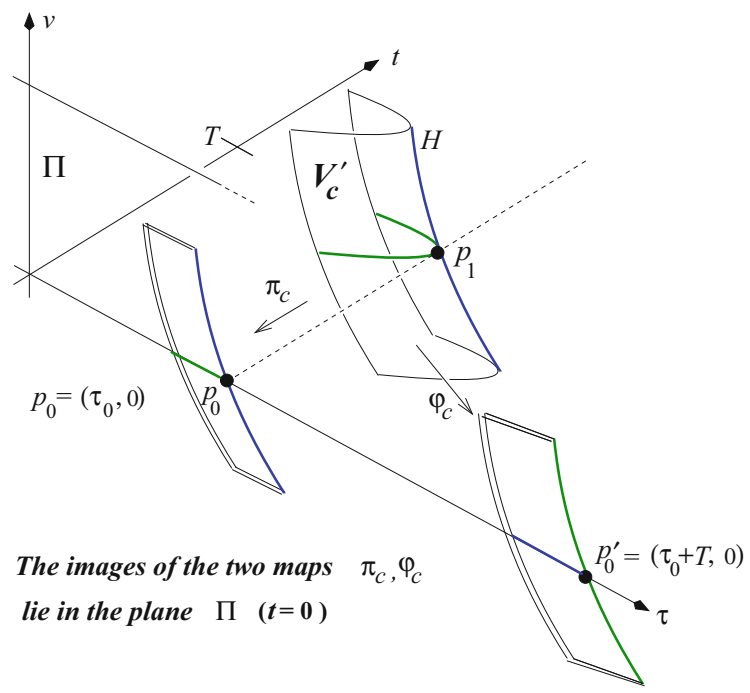

Consider now a grazing and otherwise non-impacting periodic orbit $P$ with period $T$. In terms of the impact surface the orbit $P$ is represented by a point $p_{0}=\left(\tau_{0}, 0,0\right)$ on the $\tau$-axis such that the line $l\left(\tau_{0}\right)$ through $p_{0}$ parallel to the $t$-axis intersects $V_{c}^{\prime}$ precisely at the points $p_{n}=\left(\tau_{0}, 0, n T\right), n \in \mathbf{Z}$ and is tangent to $V_{c}^{\prime}$ there. Suppose the grazing is quadratic, so that $a>0$ and $\ddot{x}_{c}>0$ at all points $p_{n}$. From Proposition 2.3 we obtain

Proposition 2.4. Each of the maps $\pi_{c}: V_{c}^{\prime} \rightarrow \Pi$ and $\phi_{c}: V_{c}^{\prime} \rightarrow \Pi$ has a fold singularity at each of the points $p_{n}, n \neq 0$.

See Fig. 2.1 for an illustration of this geometry for $n=1$.

The $T$-periodicity of $g$ means that the impact surface $V_{c}$ is invariant under translation $\tau \mapsto \tau+T$, so working modulo $T$ on the $\tau$-axis we can regard $\phi_{c}$ as taking every $p_{n} \in V_{c}^{\prime}$ to $p_{0} \in \Pi$, as does $\pi_{c}$. Therefore at each $p_{n}, n \neq 0$, we have two fold singularities

$$
\pi_{c}, \phi_{c}:\left(V_{c}^{\prime}, p_{n}\right) \rightarrow\left(\Pi, p_{0}\right)
$$

We now use this geometry to derive a criterion for the existence of impacting period$T$ orbits after perturbation based on the following elementary properties of fold maps. By smooth curve we mean a smooth arc or 1-manifold.

Lemma 2.1. Let $f:\left(\mathbf{R}^{2}, q\right) \rightarrow\left(\mathbf{R}^{2}, p\right)$ be a fold singularity with singular set $S(f)$ (a smooth curve through $q$ ) in $\mathbf{R}^{2}$; then $U(f):=f S(f)$ is a smooth curve (fold image curve) through $p$ in $\mathbf{R}^{2}$. Let $\lambda$ be a smooth curve in $\mathbf{R}^{2}$ passing through $q$, and suppose the tangent to $\lambda$ at $q$ does not coincide with the kernel of the derivative $D f(q): \mathbf{R}^{2} \rightarrow \mathbf{R}^{2}$. Then $f(\lambda)$ is a smooth curve through $p$ and is tangent to $U(f)$. 
These facts can easily be deduced from the normal form $(x, y) \mapsto\left(x, y^{2}\right)$ for a fold $\operatorname{map}\left(\mathbf{R}^{2}, 0\right) \rightarrow\left(\mathbf{R}^{2}, 0\right)$.

\subsubsection{Single Impact Period T Orbits}

Suppose now that $p \in \Pi$ represents a single-impact $T$-periodic orbit, so that $p$ is a fixed point of the dynamical map $G_{c}: \Pi \rightarrow \Pi$, that is

$$
p=G_{c}(p)=R \circ \phi_{c} \circ F_{c}(p)
$$

This can be re-expressed as

$$
\pi_{c}(q)=R \circ \phi_{c}(q)
$$

where $q=F_{c}(p) \in V_{c}^{\prime}$. Thus, rather than seek a fixed point $p$ of $G_{c}$ directly, we instead look for a point $q$ on $V_{c}^{\prime}$ that is taken to the same point under the two maps $\pi_{c}$ and $R \circ \phi_{c}$; the advantage here is that both of these maps are smooth.

Taking local coordinates $(s, v)$ on $V_{c}^{\prime}$ at $p_{1}$ (here $t=T+s$ ) we find that the maps $\pi_{c}$ and $\phi_{c}: V_{c}^{\prime} \rightarrow \Pi$ can be expressed as

$$
\begin{aligned}
& \pi_{c}:(s, v) \mapsto\left(a^{-1} v, v\right)+O(2) \\
& \phi_{c}:(s, v) \mapsto\left(s+a^{-1} v, a s+v\right)+O(2)
\end{aligned}
$$

for constant $a=\ddot{x}\left(p_{1}\right)=\ddot{x}\left(p_{0}\right)>0$. The result (2.23) is easily obtained by noting that the fold image curve is orthogonal to $\left(\frac{\partial x_{c}}{\partial \tau}, \frac{\partial x_{c}}{\partial v}\right)$ evaluated at $p_{1}$ and using the identity (2.32) below, while (2.24) follows from expressing $V_{c}^{\prime}$ locally near $p_{1}$ as a graph $\tau=\tau(t, v)$, checking that $\left(\frac{\partial \tau}{\partial t}, \frac{\partial \tau}{\partial v}\right)=\left(0, a^{-1}\right)$ at $p_{1}$ and again using (2.32).

Consequently, in order for (2.22) to be satisfied by a point $q \in V_{c}^{\prime}$ near $p_{1}$ the coordinates $(s, v)$ of $q$ must satisfy

$$
\begin{aligned}
a^{-1} v & =s+a^{-1} v+O(2) \\
v & =-r a s-r v+O(2)
\end{aligned}
$$

which for small $(s, v)$ holds only at the origin. Thus we already have the following simple but otherwise not immediately obvious result:

Proposition 2.5. Single-impact period-T orbits cannot exist arbitrarily close to a period-T grazing orbit with nonzero acceleration at the graze.

A geometric view of this fact is as follows. Equation (2.26) represents (near $p_{1}$ ) a smooth curve $\Gamma$ through $p_{1}$, namely the locus of those points $q \in V_{c}^{\prime}$ such that the $v$-coordinates of $\pi(q)$ and $R \circ \phi(q)$ coincide. Let $\Gamma^{\prime}=\pi_{c}(\Gamma)$ and $\Gamma^{\prime \prime}=R \circ \phi_{c}(\Gamma)$ 


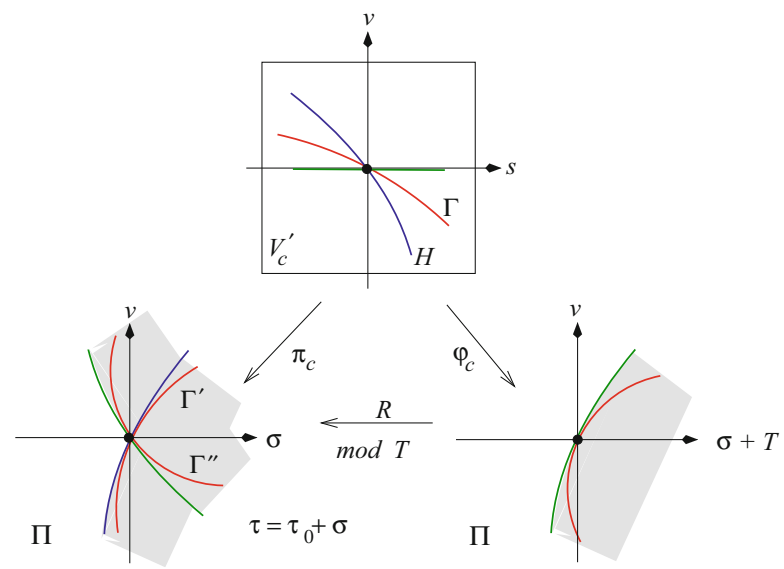

Fig. 2.2 The images $\Gamma^{\prime}$ and $\Gamma^{\prime \prime}$ of $\Gamma$ under the two fold maps $\pi_{c}$ and $R \circ \phi_{c}$ modulo $T$

be the respective images of $\Gamma$ : then $\pi_{c}(q)=R \circ \phi_{c}(q)$ precisely when the curves $\Gamma^{\prime}$ and $\Gamma^{\prime \prime}$ intersect at that point. Now since $\Gamma$ is tangent neither to the kernel of $D \pi_{c}\left(p_{1}\right)$ (the $s$-axis) nor the kernel of $D \phi_{c}$ at $p_{1}$ (which is spanned by $(a,-1)$ ) it follows from Lemma 2.1 that $\Gamma^{\prime}$ and $\Gamma^{\prime \prime}$ are tangent to the fold image curves of $\pi_{c}$ and $R \circ \phi_{c}$, respectively. However, (2.23) and (2.24) show that these fold curves are tangent to the directions $(1, a)$ and $(1,-r a)$, respectively, and so with $\tau$ reduced modulo $T$ these curves clearly intersect only at $(\tau, v)=\left(\tau_{0}, 0\right)$. See Fig. 2.2.

From this picture it is now easy to visualize the effect of a smooth perturbation to the system, parametrized by a scalar parameter $\mu$. We suppose that $f$ and $g$ in (2.1) are replaced by $f_{\mu}$ and $g_{\mu}$ (with $c$ also possibly replaced by $c_{\mu}$ ) taking their original values at $\mu=0$. The stability of fold singularities ensures that for sufficiently small $|\mu|$ there exists a unique point $p_{1}(\mu)$ on $V_{c}^{\prime}(\mu)$, varying smoothly with $\mu$, which is a fold singularity point for both $\pi_{c}, \phi_{c}: V_{c}^{\prime}(\mu) \rightarrow \Pi$ with $c=c_{\mu}$. Let $k=k(\mu)$ and $l=l(\mu)$ denote the $\tau$-coordinate of the image of $p_{1}(\mu)$ under $\pi_{c}, \phi_{c}$ respectively, so that the equations of the tangent lines to the fold image curves of $\pi_{c}, \phi_{c}$ through $(k, 0),(l, 0)$ are

$$
\begin{aligned}
& v=m(\tau-k) \\
& v=n(\tau-l)
\end{aligned}
$$

with slopes $m=m(\mu)$ and $n=n(\mu)$ that are close to $a$ and $-r a$, respectively, for small $|\mu|$. It is elementary to check that the $v$ coordinate of the point of intersection of the two lines (2.27), (2.28) is positive (so that a single impact $T$-periodic orbit occurs locally) if and only if $k-l$ has the same sign as $\left(\frac{1}{n}-\frac{1}{m}\right)$. Since for $\mu=0$ we have $\left(\frac{1}{n}-\frac{1}{m}\right)=-a^{-1}\left(r^{-1}+1\right)<0$, the criterion becomes simply that $l-k>0$ for sufficiently small $|\mu|$. In the present context by construction $k=\tau$ and $l=\tau+t-T$ and so we arrive at the following result. 
Proposition 2.6. A single-impact T-periodic orbit occurs for sufficiently small $|\mu| \neq 0$ if and only if the t-coordinate $t_{1}(\mu)$ of $p_{1}(\mu)$ is greater than $T$.

In other words, there is a $T$-periodic impacting orbit provided that the unique nearby initially grazing orbit that grazes a second time takes longer than time $T$ to do so.

From the definition of $p_{1}(\mu)$ as the unique point close to $p_{1}$ which satisfies $x_{c}=\dot{x}_{c}=0$ as well as $v=0$ we find from implicit differentiation that

$$
t_{1}^{\prime}(0)=-a^{-1}\left(\frac{\partial x_{c}}{\partial \tau}\right)^{-1} \operatorname{det} W
$$

where $W$ denotes the matrix

$$
\left(\begin{array}{ll}
\frac{\partial x_{c}}{\partial \tau} & \frac{\partial x_{c}}{\partial \mu} \\
\frac{\partial \dot{x}_{c}}{\partial \tau} & \frac{\partial \dot{x}_{c}}{\partial \mu}
\end{array}\right)
$$

evaluated at $p_{1}(0)$. If we assume $t_{1}^{\prime}(0) \neq 0$ then since $a>0$ the necessary and sufficient condition for the existence of a single-impact $T$-periodic orbit close to grazing becomes explicitly:

$$
\left(\frac{\partial x_{c}}{\partial \tau}\right)^{-1} \operatorname{det} W \mu<0 .
$$

At first sight this appears distinct from Nordmark's criterion in Proposition 2.1 which involves derivatives with respect to $v$ (the terms $\gamma, \delta$ ) rather than with respect to $\tau$. The two are reconciled, however, using the fact that, regarding the system (without the obstacle) as generating a flow $\left\{\Psi_{t}\right\}$ in $(x, \dot{x}, \tau)$-space, the Jacobian matrix $D \Psi_{t}(c, v, \tau)$ takes the initial tangent vector $(v, a, 1)^{t}$ to the time- $t$ tangent vector $(\dot{x}, \ddot{x}, 1)^{t}$. For a grazing periodic orbit of period $T$ this means that $(v, a, 1)^{t}$ is an eigenvector of $D \Psi_{T}(c, 0, \tau)$ with eigenvalue 1, giving the identities

$$
\begin{aligned}
& \frac{\partial x_{c}}{\partial v} a+\frac{\partial x_{c}}{\partial \tau}=0 \\
& \frac{\partial \dot{x}_{c}}{\partial v} a+\frac{\partial \dot{x}_{c}}{\partial \tau}=a
\end{aligned}
$$

with derivatives evaluated at $p_{0}$ (or $p_{1}$ ). Using these we find that

$$
\operatorname{det} W=-a((1-\delta) \kappa+\gamma \lambda)=-a \psi
$$

in the notation of Sect. 2.1.1. Thus the criterion (2.30) becomes $\gamma \psi<0$ just as in Proposition 2.1.

Observe that here we have not required the condition $\operatorname{det}(I-A) \neq 0$, and so (in contrast to [13] where this is heavily used) we have no information about the persistence of a non-impacting $T$-periodic orbit for $|\mu| \neq 0$. 


\subsubsection{Single Impact 2T-Periodic Orbits}

We next apply the impact surface geometry to the criterion for persistence of a $2 T$-periodic single-impact orbit. Clearly there are two criteria that together are necessary and sufficient for persistence of a $2 T$-periodic single-impact orbit, namely:

1. The criterion analogous to (2.30) at $p_{2}(0)$ rather than $p_{1}(0)$

2. The condition that $V_{c}^{\prime}$ does not intervene between $p_{0}(\mu)$ and $p_{2}(\mu)$.

To formulate the second criterion, we need information not only about the positions of fold image curves in $\Pi$ but also about which side of such a curve is the one that lies in the image of the fold map. From the normal form $(x, y) \mapsto\left(x, y^{2}\right)$ for a fold map we deduce that a coordinate-independent description is as follows.

Lemma 2.2. Let $f:\left(\mathbf{R}^{2}, q\right) \rightarrow\left(\mathbf{R}^{2}, p\right)$ be a fold singularity as in Lemma 2.1. Let $u \in K=\operatorname{ker} D F(q)$ be a nonzero vector. The restriction $f \mid(q+K)$ has the form

$$
f: q+\xi u \mapsto p+\xi^{2} w+O\left(\xi^{3}\right)
$$

for a nonzero vector $w \in \mathbf{R}^{2}$ that does not lie in the range of $D f(q)$. Thus $p+\eta w$ lies in the range of $f$ for all sufficiently small $\eta>0$.

In our case we have that at every point $p_{n}(n>0)$ the kernel $K_{\pi}$ of $D \pi_{c}\left(p_{n}\right)$ is the $s$-axis while the kernel $K_{\phi}$ of $D \phi_{c}\left(p_{n}\right)$ is spanned by the vector $(1,-a)$, using local coordinates $(s, v)$ on $V_{c}^{\prime}$ near $p_{n}$ as in (2.24). Moreover, representing $V_{c}^{\prime}$ locally as the graph of a smooth function $\tau=\tau(s, v)$ we find by implicit differentiation of (2.20) that at each such $p_{n}$

$$
\frac{\partial^{2} \tau}{\partial t^{2}}=-a\left(\frac{\partial x_{c}}{\partial \tau}\right)^{-1}=\left(\frac{\partial x_{c}}{\partial v}\right)^{-1}=: \gamma_{n}^{-1}
$$

using (2.31), and also (with a little more effort) that

$$
(a,-1) \cdot D^{2} \phi_{c} \mid K_{\phi}: \xi(-1, a) \mapsto-\xi^{2} a \gamma_{n}^{-1} \operatorname{det} A^{n}=-k \gamma_{n}^{-1}
$$

for $k>0$, where the dot denotes scalar product. Using this information, sketches of the images of the fold maps $\pi_{c}, \phi_{c}$ close to $p_{2}$ and the map $\pi_{c}$ close to $p_{1}$ quickly indicate that the condition for $V_{c}^{\prime}$ not to intervene between $p_{0}(\mu)$ and $p_{2}(\mu)$ is

$$
\left(t_{1}^{\prime}(\mu)-t_{2}^{\prime}(\mu)\right) \gamma>0
$$

where $\gamma=\gamma_{1}$ by definition.

The point $p_{n}(\mu)=\left(\tau_{n}(\mu), 0 ; t_{n}(\mu)\right)$ is obtained by solving

$$
x_{c}(\tau, 0 ; t, \mu)=\dot{x}_{c}(\tau, 0 ; t, \mu)=0
$$


close to $p_{n}=p_{n}(0)$, from which we find

$$
\left(\begin{array}{cc}
\frac{\partial x_{c}}{\partial \tau} & 0 \\
\frac{\partial \dot{x}_{c}}{\partial \tau} & a
\end{array}\right)\left(\begin{array}{c}
\tau_{n}^{\prime}(0) \\
t_{n}^{\prime}(0)
\end{array}\right)=-\left(\begin{array}{c}
\frac{\partial x_{c}}{\partial \mu} \\
\frac{\partial \dot{x}_{c}}{\partial \mu}
\end{array}\right)
$$

with partial derivatives evaluated at $p_{n}$, giving

$$
\tau_{n}^{\prime}(0)=-\left(\frac{\partial x_{c}}{\partial \tau}\right)_{n}^{-1} \kappa_{n}=a^{-1} \gamma_{n}^{-1} \kappa_{n}
$$

with the obvious notation and assuming as before that $\gamma$ and $\eta$ (hence also $\gamma_{2}$ by (2.15)) are nonzero. The criterion (2.33) therefore becomes

$$
\kappa \mu>\gamma_{2}^{-1} \gamma \kappa_{2} \mu
$$

From the identity

$$
\kappa_{2}=(\alpha+1) \kappa+\gamma \lambda
$$

this becomes

$$
\kappa \mu>\eta^{-1}((\alpha+1) \kappa+\gamma \lambda) \mu
$$

that is (since a number has the same sign as its inverse)

$$
\eta((1-\delta) \kappa+\gamma \lambda) \mu<0
$$

For the criterion (1) the counterpart of (2.30) at $p_{2}$ is

$$
\left(\frac{\partial x_{c}}{\partial \tau}\right)_{2}^{-1} \operatorname{det} W_{2} \mu<0,
$$

where $W_{2}$ is the matrix (2.1.3) evaluated at $p_{2}$. Using the easily verified fact that $W_{2}=(I+A) W$, we may rewrite this as

$$
\eta^{-1} \gamma^{-1} \Delta_{+}((1-\delta) \kappa+\gamma \lambda) \mu<0
$$

From (2.37) we see that the additional condition for criterion (1) to hold is that $\gamma \Delta_{+}>0$, and so we recover Proposition 2.2.

\subsubsection{Conclusion}

We have shown how the impact surface approach to the geometry of grazing bifurcation allows an easy derivation of criteria for the existence of single-impact 
$T$-periodic orbits or $2 T$-periodic orbits close to grazing for a $T$-periodically forced impact oscillator. As in the study by Nordmark [13], the methods can naturally be extended to $n T$-periodic orbits with $m$ impacts. However, a further advantage of the impact surface description is that the tools of singularity theory can be applied in this context to study multiparameter bifurcation of periodic orbits close to degenerate grazing ( $a=0)$ where the geometry of the relevant singularities (described in [5]) is more complicated than the fold geometry that arises here. We aim to pursue this study in further work.

\section{References}

1. di Bernardo, M., Budd, C.J., Champneys, A.R., Kowalczyk, P.: Piecewise-Smooth Dynamical Systems: Theory and Applications. Springer, Berlin (2008)

2. di Bernardo, M., Budd, C.J., Champneys, A.R., Kowalczyk, P., Nordmark, A.B., Tost, G.O., Piiroinen, P.T.: Bifurcations in nonsmooth dynamical systems. SIAM Rev. 50, 629-701 (2008)

3. Brogliato, B.: Nonsmooth Mechanics. Springer, London (1999)

4. Budd, C.J., Dux, F.J., Cliffe, A.: The effect of frequency and clearance variations on singledegree-of-freedom impact oscillators, J. Sound Vib. 184, 475-502 (1995)

5. Chillingworth, D.R.J.: Discontinuity geometry for an impact oscillator. Dyn. Syst. 17, 389-420 (2002)

6. Chin, W., Ott, E., Nusse, H.E., Grebogi, C.: Grazing bifurcation in impact oscillators. Phys. Rev. E 50, 4427-4444 (1994)

7. Dankowicz, H., Jerrelind, J.: Control of near-grazing dynamics in impact oscillators. Proc. R. Soc. Lond. A 461, 3365-3380 (2005)

8. Ivanov, A.P.: Impact oscillations: linear theory of stability and bifurcations. J. Sound Vib. 178, 361-378 (1994)

9. Küpper, T., Hosham, H.A., Weiss, D.: Non-smooth dynamical systems via reduction methods. In: Johann, A. (ed.) Recent Trends in Dynamical Systems. Springer Proceedings in Mathematics \& Statistics 2013

10. Molenaar, J., de Weger, J.G., van de Water, W.: Mappings of grazing-impact oscillators. Nonlinearity 14, 301-321 (2001)

11. Nordmark, A.B.: Non-periodic motion caused by grazing incidence in an impact oscillator. J. Sound Vib. 145, 279-297 (1991)

12. Nordmark, A.B.: Universal limit mapping in grazing bifurcations. Phys. Rev. E 55, 266-270 (1997)

13. Nordmark, A.B.: Existence of periodic orbits in grazing bifurcations of impacting mechanical oscillators. Nonlinearity 14, 1517-1542 (2001)

14. Stewart, D.: Rigid-body dynamics with friction and impact. SIAM Rev. 42, 3-39 (2000)

15. Whiston, G.W.: Singularities in vibro-impact dynamics. J. Sound Vib. 152, 427-460 (1992)

16. Zhao, X., Dankowicz, H.: Unfolding degenerate grazing dynamics in impact actuators. Nonlinearity 19, 399-418 (2006) 